Eur J Clin Chem Clin Biochem

$1995 ; 33: 855-858$

(c) 1995 Walter de Gruyter \& Co. Berlin · New York

\title{
Creatinine and Surveys: An Assessment
}

\section{By Bert G. Blijenberg', Rita J. Brouwer', Henk Baadenhuijsen ${ }^{2}$ and Geert J. M. Boerma ${ }^{3}$}

1 Department of Clinical Chemistry, University Hospital Rotterdam-Dijkzigt, Rotterdam, The Netherlands

${ }^{2}$ Department of Clinical Chemistry, University Hospital Nijmegen-St. Radboud, Nijmegen, The Netherlands

3 Department of Clinical Chemistry, University Hospital Rotterdam-Sophia Children's Hospital, Rotterdam, The Netherlands

(Received May 15/July 21, 1995)

Summary: We analysed the results of surveys on creatinine held in The Netherlands during the years 1992, 1993 and 1994. Assay results of 113 samples were reviewed: 88 human sera and 25 samples of animal origin. The results of 5 creatinine assays, 4 based on the Jaffé reaction and 1 enzymatic procedure, are discussed.

The enzymatic assay showed by far the best performance, while some of the Jaffé methods differed considerably. All results were evaluated by reference to a HPLC-based selected method for creatinine.

Our study shows the need for caution when applying survey performance criteria for creatinine.

\section{Introduction}

In two earlier publications we described the influence of a number of factors on the accuracy of various creatinine assays developed for routine use in clinical chemistry (1, 2). We also questioned the value of quality assessment specimens for creatinine assays.

Since these earlier studies provided only limited data on accuracy-influencing factors and the value of quality assessment specimens, and in view of the large variation of results, especially in some modifications of the Jaffé reaction, we decided to study these aspects in greater detail in a wider setting.

Here, we describe the cooperation. between three laboratories, all participating in the serum chemistry section of the Dutch Quality Assessment Foundation (SKZL) (3). The results submitted during the years 1992, 1993 and 1994 were compared with those obtained with our high performance liquid chromatography (HPLC) based selected method for creatinine (4).

In addition, all samples under study were analysed separately with the instruments also used in our previ- ous study i.e. the DuPont Dimension and the Merck ELAN.

\section{Materials and Methods}

Materials

One hundred and thirteen control sera were included in this study, 25 of animal origin, 88 of human origin. During the years 1992,

Tab. 1 Regression equations of all comparisons

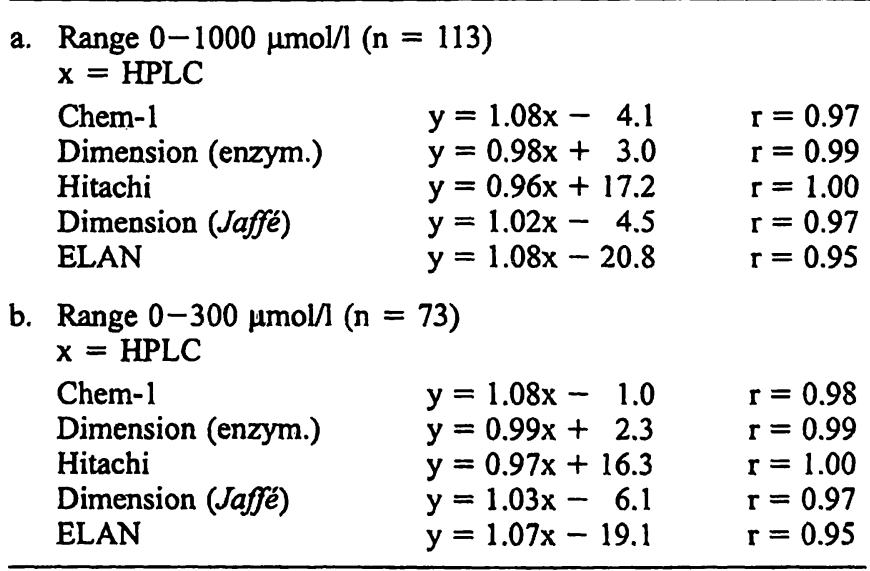


1993 and 1994 they were distributed in the normal way by the official Quality Assessment Foundation in The Netherlands to all participating laboratories.

\section{Methods}

Five routine methods for creatinine were applied with the following instruments:

1. Bayer-Technicon Chem-1 with a Jaffé reaction based method

2. Boehringer Mannheim Hitachi 717 and 747 with a Jaffé reaction based method

3. DuPont Dimension with an enzymatic method
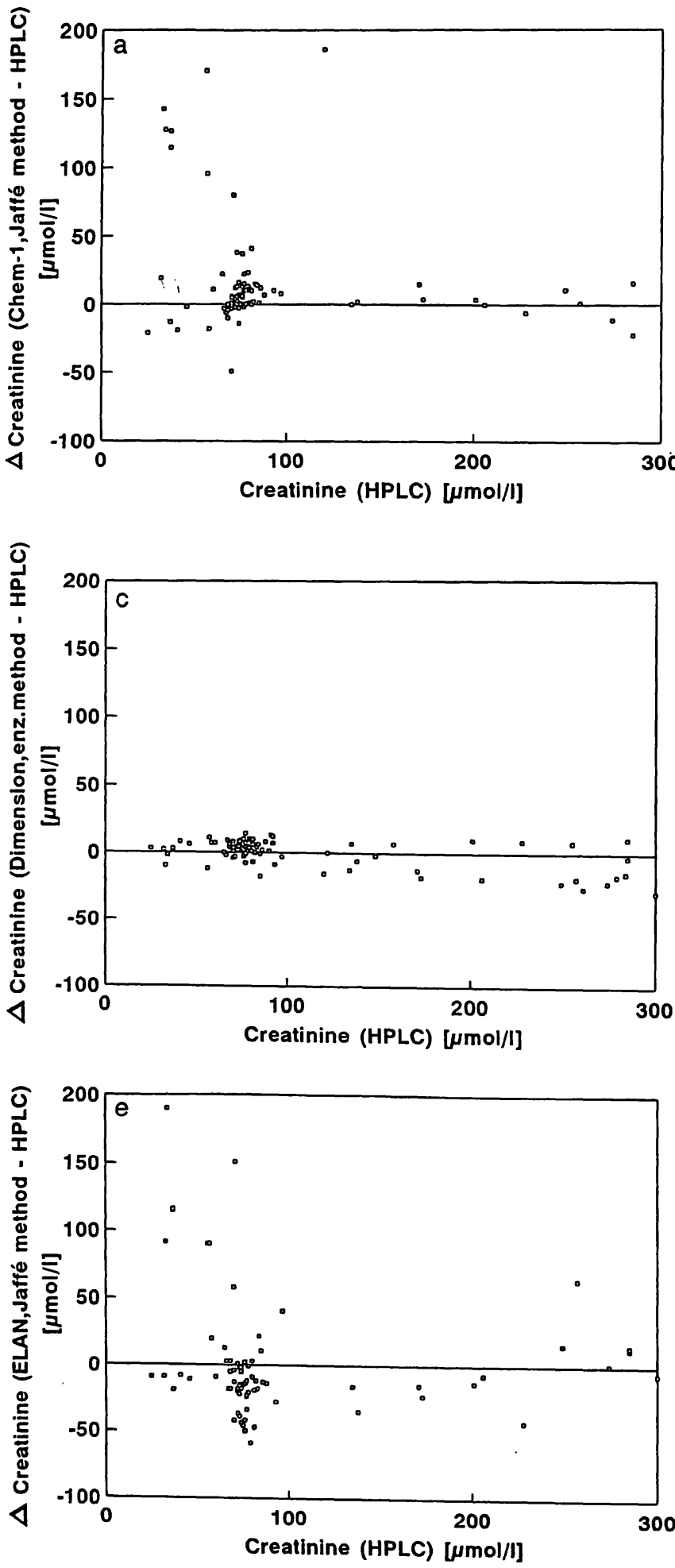

\section{DuPont Dimension with a Jaffé reaction based method}

5. Merck ELAN with a Jaffé reaction based method

Methods 1, 2, 4 and 5 were routine procedures as supplied by the various manufacturers. Method 3 is a home made adaptation of the Boehringer Mannheim enzymatic method (creatininase) to the Dimension (5). All instruments were run according to the manufacturers' instructions.

\section{Procedure}

The results of methods 1,2 and 3 were taken from the quality control files of the three participating laboratories. Similarly, the
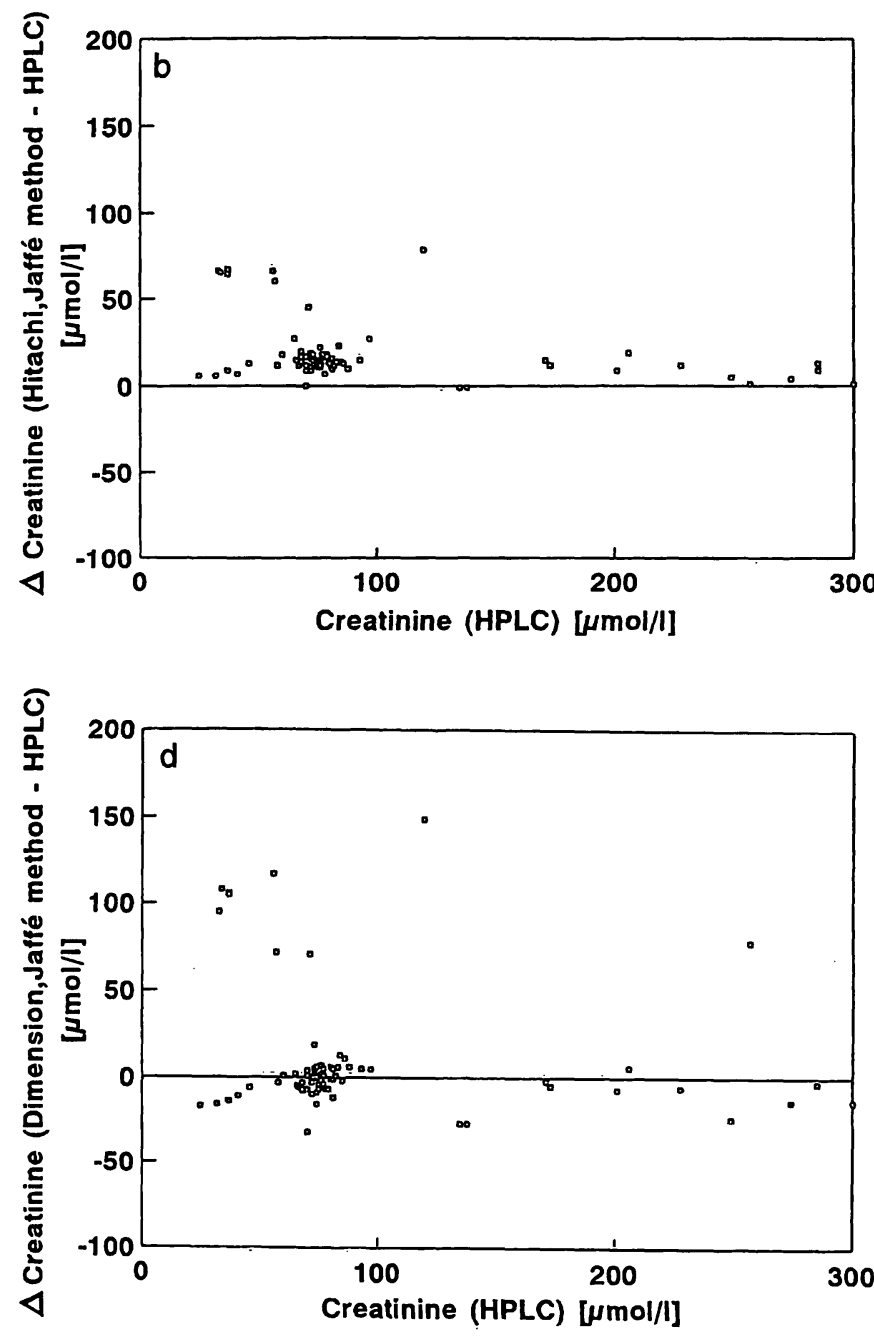

Fig. 1 Residual creatinine values for the various methods (see section Methods) obtained with human control samples with creatinine (HPLC) concentrations lower than' $300 \mu \mathrm{mol} / \mathrm{l}$. 
HPLC results were taken from the Academic Hospital Rotterdam, which is part of the National External Quality Assessment Scheme.

Extra samples were stored at $-80^{\circ} \mathrm{C}$ during 1992,1993 and 1994 , and used for the methods 4 and 5 .

\section{Statistical analysis}

Regression analysis was done according to Passing \& Bablok (6).

\section{Results}

We started by comparing the routine methods (methods 1, 2 and 3) with the HPLC selected method. All values deviating more than $20 \%$ from the corresponding HPLC result were redetermined. This figure of $20 \%$ was chosen arbitrarily. In total 7 results proved to be clerical errors.

Then, using the same set of specimens, creatinine was determined on the Dimension (Jaffé method) and the ELAN.

All regression equations are tabulated separately in table 1 .

In view of the clinical significance of low to medium range creatinine concentrations, those samples of human origin with HPLC values lower than $300 \mu \mathrm{mol} / 1$ were plotted separately (figs. 1a-e).

The regression equations for the range $0-300 \mu \mathrm{mol} / \mathrm{l}$ are also included in table 1.

\section{Discussion}

Our results from the elaborated study generally confirm those found earlier, thus providing a sounder basis for evaluation. The enzymatic procedure for creatinine scores better than the Jaffé reaction based assays with the specimens used in the Dutch Quality Assessment Scheme. There are also differences between the various creatinine (Jaffé) methods.

Many samples showing deviations from the HPLC results are problematic for all Jaffé reaction based creatinine methods. Nevertheless, it is possible to construct a worst-case scenario by comparing the Chem-1 data with the ELAN, as in figure 2.

We assume that the differences in the results of the various Jaffé methods are due to the assay design. They are all kinetic measurements, but they differ considerably with respect to sample and reagent volumes, reagent concentrations and choice of reagents and type of measurement (mono- vs. bichromatic and timing of absorbance readings). The ELAN does not employ a bilirubin correction, the Hitachi and Chem-1 have chromatic cor-

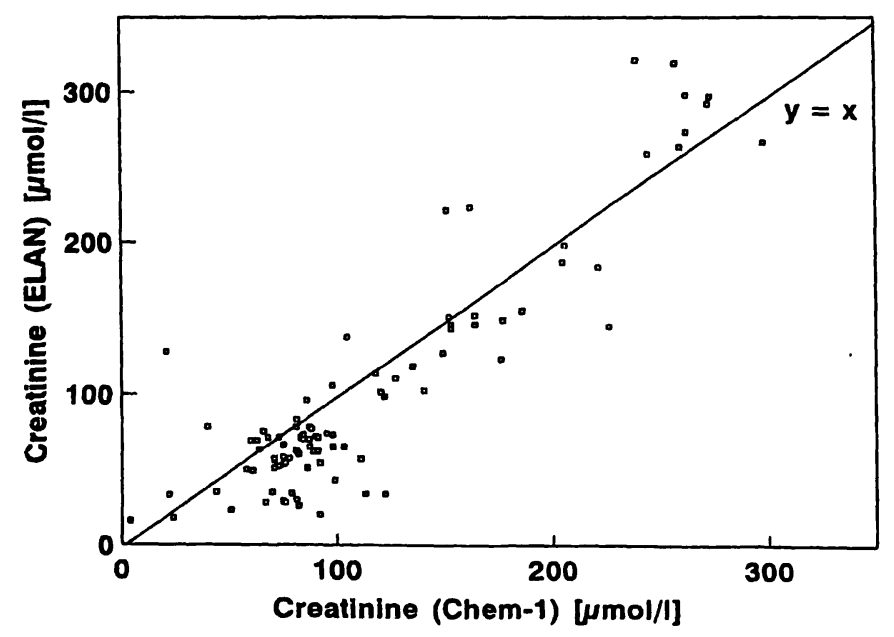

Fig. 2 Graphical presentation of human control serum results obtained with the ELAN and the Chem-1.

Regression equation: $y=1.11 x-30.0 ; r=0.89$

$\mathrm{x}$ (mean) $=93 \mu \mathrm{mol} / \mathrm{l}, \mathrm{y}($ mean $)=110 \mu \mathrm{mol} / \mathrm{l}$

$x($ median $)=87 \mu \mathrm{mol} / \mathrm{l}, \mathrm{y}($ median $)=66 \mu \mathrm{mol} / 1$

rections, while the Dimension reagent contains potassium hexacyanoferrate(III) for bilirubin oxidation. Due to the influence of bilirubin, the ELAN showed considerable deviations from the HPLC values, especially with two extreme specimens containing 222 and 343 $\mu \mathrm{mol}$ bilirubin per litre; specimens with bilirubin values of about $100 \mu \mathrm{mol} / \mathrm{l}$ (6 in total) also showed discrepant creatinine values.

Most of the deviating results for all methods, however, could not be attributed to interference by bilirubin. Most of the human serum samples were pooled left-over patient samples, sometimes spiked with additional material like enzymes, bilirubin and sucrose. This makes it nearly impossible to understand the cause of the deviation. However, the findings with these human samples do show again the limitations of the Jaffe reaction based creatinine methods in the daily routine of a laboratory. It is also clear from figure 2 that the expression "Jaffé method" is an inaccurate statement.

The commercial sera used, BioRad (Lyphochek and Liquichek) and Beckman Decision, show an interesting pattern. The various techniques showed particularly substantial differences in the analysis of BioRad products, so that the use of these products in surveys may be questionable. Of course, this argument does not hold for the normal application of these products in precision checks of a procedure.

These results reveal once more the general difficulty with most classical External Quality Assessment (EQA) schemes. Issues of cost, stability and available analyte concentration often necessitate the use of processed lyophilized control serum, which very often lack the required degree of commutability with the native human serum specimens. As correctly pointed out by Thienpont 
and Stöckl (7, 8), present-day quality assessment schemes expose the dilemma of which combination of control material and target value to choose. This dilemma is particularly acute when the results of such schemes are to be used for accreditation/licensing purposes.

Summarizing, the enzymatic method for creatinine used in the present survey scores higher than any of the Jaffé reaction based methods, based on comparison with our HPLC based selected method. For the Jaffe reaction based methods, survey material must be chosen care-

\section{References}

1. Blijenberg BG, Brouwer HJ, Kuller TJ, Leeneman R, Leeuwen CJM van. Improvement in creatinine methodology: a critical assessment. Eur J Clin Chem Clin Biochem 1994; 32:529-37.

2. Blijenberg BG, Brouwer HJ. The accuracy of creatinine methods based on the Jaffé reaction: a questionable matter. Eur $\mathrm{J}$ Clin Chem Clin Biochem 1994; 32:909-13.

3. Steigstra $H$, Jansen RT, Baadenhuijsen $H$. Combi scheme: new combined internal/external quality-assessment scheme in The Netherlands. Clin Chem 1991; 37:1196-204.

4. Zwang L, Blijenberg BG. Assessment of a selected method for creatinine with special emphasis on bilirubin interference. Eur $\mathrm{J}$ Clin Chem Clin Biochem 1991; 30:779-84.

5. Guder WG, Hoffmann GE, Hubbuch A, Poppe WA, Siedel J, Price CP. Multicentre evaluation of an enzymatic method for creatinine determination using a sensitive colour reagent. J Clin Chem Clin Biochem 1986; 24:889-902. fully and possibly selectively. Results from samples of animal origin have not been described in detail, because of the limited number used $(n=25)$; however, the results were generally similar to those obtained with the human serum samples.

$$
: 1
$$

\section{Acknowledgements}

The authors wish to thank Mrs. A. P. Copper-Staamer for clerical support and Mr. R. Leeneman (Rotterdam) and Mrs. M. Hessels (Nijmegen) for help with data collection.

6. Passing $\mathrm{H}, \mathrm{Bablok} \mathrm{W}$. A new biomedical procedure for testing the equality of measurements from two different analytical methods. J Clin Chem Clin Biochem 1983; 21:709-20.

7. Stöckl D, Thienpont LM. The combined-target approach: a way out of the proficiency testing dilemma. Arch Pathol Lab Med 1994; 118:775-6.

8. Thienpont LM, Stöckl D. External quality assessment with accuracy-based kits: bringing together consensus/reference method targets and method dependent targets. Ann Clin Biochem. In press.

Dr. B. G. Blijenberg Academic Hospital Rotterdam-Dijkzigt Department of Clinical Chemistry

Dr. Molewaterplein 40

NL-3015 GD Rotterdam

The Netherlands 